\title{
An Agent Based Model of the Nash Demand Game in Regular Lattices
}

\author{
David Poza $^{1}$, José Manuel Galán ${ }^{2}$, José Ignacio Santos², and Adolfo López-Paredes ${ }^{1}$ \\ ${ }^{1}$ Grupo INSISOC, Dpto. de Organización de Empresasy CIM, Escuela de Ingenierías \\ Industriales, Universidad de Valladolid, Pso del Cauce S/N, 47011 Valladolid \\ ${ }^{2}$ Grupo INSISOC, Área de Organización de Empresas, Dpto. de Ingeniería Civil, Escuela \\ Politécnica Superior, Universidad de Burgos, Edificio La Milanera, C/ Villadiego S/N, \\ 09001 Burgos \\ djpoza@gmail.com, \{jmgalan,jisantos\}@ubu.es, adolfo@insisoc.org
}

\begin{abstract}
In this work we propose an agent based model where a fixed finite population of tagged agents play iteratively the Nash demand game in a regular lattice. This work extends the multiagent bargaining model by [1] including the spatial dimension in the game. Each agent is endowed with memory and plays the best reply against the opponent's most frequent demand. The results show that all the possible persistent regimes of the global interaction game can also be obtained with this spatial version. Our preliminary analysis also suggests that the topological distribution of the agents can generate new persistent regimes within groups of agents with the same tag.
\end{abstract}

Keywords: Agent-based modeling, Nash demand game, game theory, negotiation, segregation, tags, social norms.

\section{Introduction}

The gist of agent-based modeling consists in the individual abstraction of the entities that participate in a given target system as computational entities - agents - in a model [2], [3]. It also facilitates to establish a direct correspondence between the interactions observed as agent's interactions, and hence to analyze the emerging behavior.

In the last twenty years, this technique has become popular in almost every scientific domain, such as natural resources management [4], biology [5], project management [6], etc. Nonetheless, it has been particularly useful in economic and social systems as a formalization tool.

The role that norms play as regulator mechanisms of certain aspects of social, economic and organizational behaviors has been thoroughly studied in social sciences [7], and even by means of agent-based modeling [8]. Once a norm has been established, it acts as a self-reinforcement mechanism of behavior. However, the emergence of norms is, in general, exempt from explicit mechanisms of control. This is a key factor to understand the informal behavior of the organizations.

In this work, we analyze the emergence of norms using the agent-based model proposed by [1], AEY's model from now on, which is an extension of the well known 
Nash demand game. By studying the transient and the asymptotic dynamics of this model, Axtell et al proved that self-reinforced norms can emerge spontaneously. These emergent norms may be completely different from one another even though all the agents of the population have the same behavior rule. Moreover, when agents are endowed with a tag (which initially has no meaning) and remember the past behaviors of their opponents and their tags, it is possible to demonstrate that segregation, in terms of different behavior within and outside a group, can emerge endogenously.

We have generalized the original model by adding a new behavior rule that requires less cognitive abilities than those required in the original paper. Furthermore, when agents used this new behavior rule, the segregation emerged even more frequently. In our analysis, we have also incorporated the influence of the topology on the results of the game. To this aim, we have considered the spatial dimension of the game by introducing a regular spatial structure.

This work is organized as follows: first, we will briefly explain the extensions and modifications that we have performed to generalize the AEY original model. Next, we will describe the results that we have obtained when agents are randomly distributed. Afterwards, we will discuss some cases where several persistent regimes can simultaneously emerge and the possible relation with mesoscopic topological effects. We will finish with conclusions and extensions of this work.

\section{AEY's Model in Regular Spatial Structures}

In AEY's model there is a population of $n$ agents that repetitively play a bargaining game. The interaction process in the model consists in the following: two agents randomly selected from the population of agents - demand some portion of a pie (which is a metaphor of something that is going to be shared between two persons). The portion of the pie that each agent gets depends on the corresponding portion demanded by her opponent: they get what they demand as long as the sum of both demands is not higher than the whole of the pie; otherwise both get nothing.

In order to simplify the analysis we will assume that agents have only three possible demands: low (30\%), medium (50\%) and high (70\%). Table 1 shows one agent's reward depending on her opponent's demand -[9] study the influence of payoffs in the game-.

Table 1. Payoff matrix

\begin{tabular}{|c|c|c|c|}
\hline & $\mathrm{H}$ & $\mathrm{M}$ & $\mathrm{L}$ \\
\hline $\mathrm{H}$ & 0,0 & 0,0 & 70,30 \\
\hline $\mathrm{M}$ & 0,0 & 50,50 & 50,30 \\
\hline $\mathrm{L}$ & 30,70 & 30,50 & 30,30 \\
\hline
\end{tabular}

Agents have a memory in which they keep the latest $m$ decisions taken by their opponents in previous games (where $m$ is the memory length). Agents check their memory and take their decision depending on the strategies followed by their opponents in the previous matches. When the game starts, the agents' memories are initialized with $\mathrm{m}$ random values between the three options they can demand (low, medium or high). 
There is a small probability of a player not following the rational decision rule and playing a random strategy instead, called the mutation probability $\varepsilon$. In the most sophisticated version of the model, each agent can be identified as a member of one of the two possible groups by means of a tag: agents store in their memory their opponents' demands conditioned by the corresponding group.

In our simulation model, agents take decisions by using two different decision rules: either maximizing the expected utility in function of the memory vector, or demanding the best reply against the opponent's most frequent demand.

In the original model by AEY, players randomly paired at each tick could play with any other in the population. However, in our spatial version of the model where agents are placed on a 10x10 toroidal reticule, each one interacts with one of her eight spatial neighbours located in a radius-1 Moore neighbourhood.

A simulation run stops when the system reaches a persistent regimen. As there are no absorbent states for $\varepsilon>0$, we consider that a persistent regimen has been reached either when all the agents have at least $(1-\varepsilon) \cdot \mathrm{m}$ instances of 'medium' behaviour in their memories (an equitable norm) or when there are, at most, $\varepsilon \cdot \mathrm{m}$ instances of 'medium' behaviour in their memories (a non-equitable norm). When tags are considered in the game, the stop criterion is applied to both agents' memories corresponding to same and different tagged opponents.

\section{Equilibrium in a Random Scenario}

We have studied the model in different scenarios. In the first one, tagged agents are randomly distributed in the grid (see Figure 1).

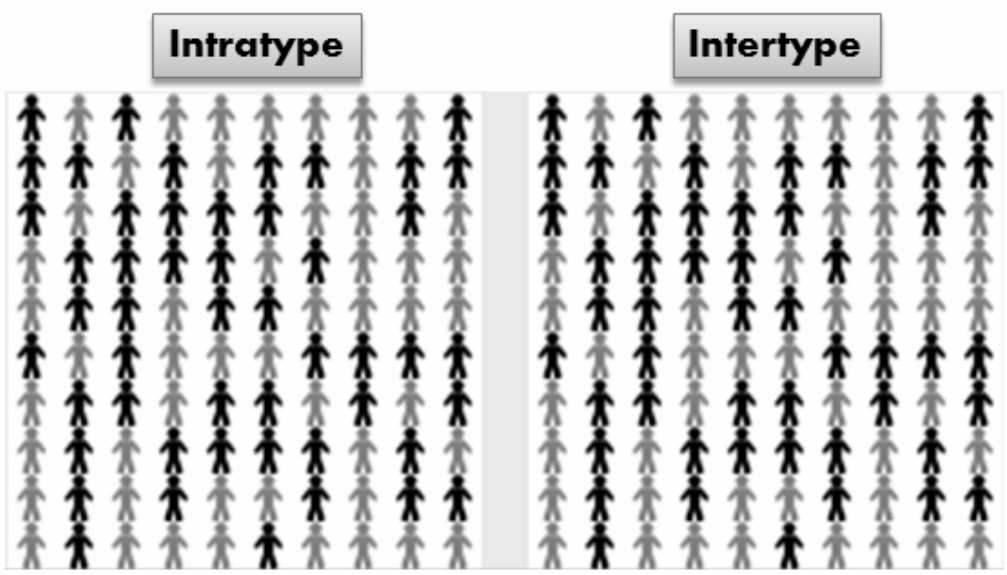

Fig. 1. A sample of random distribution of agents in the grid. In order to show clearly the players' strategies for both kind s of contacts, two symmetric grids are used to represent interactions between agents with the same tag (the left one) and with different tag (the right one), called "intratype" and "intertype" interactions respectively. 
The system is an ergodic time-homogeneous Markov chain [10]. Although there is a strictly positive probability of finding the system in each of its states in the long run, some states are more persistent than others -the probability of staying in them is higher-. The best way of representing the state space is a simplex of the agents' memory states. Because the memory of an agent is made by two partitions, corresponding to the past demands of the two classes of opponents, we use two simplexes to represent both ones.

The pure strategy equilibrium MM of the Nash demand game, or equity norm, is a stochastically stable equilibrium for mutation rates small enough -once it is reached, it is very unlikely the system leaves it-. However, other regions of inequality strategies can be considered as pseudo-stable because the transition time to go from them to the equity norm is enormously long, and it grows exponentially with the number of agents and the memory length $\mathrm{m}$, so the system can permanence in them over a long time.
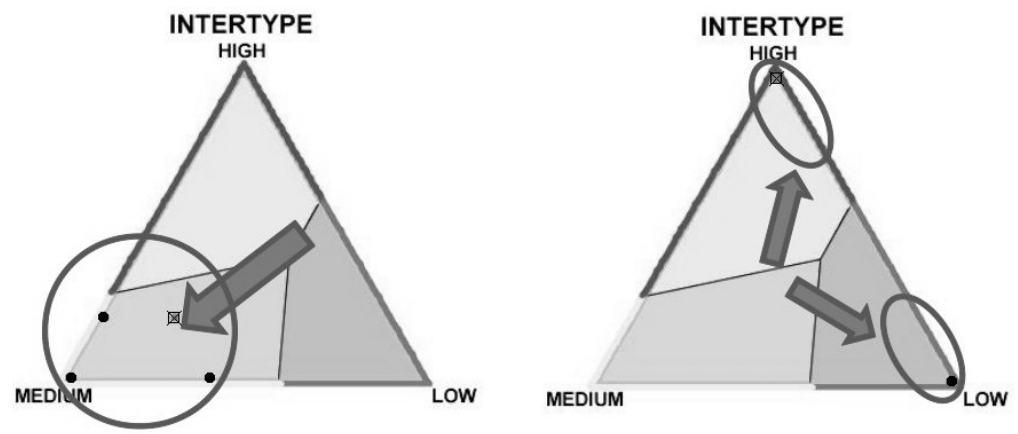

Fig. 2. Most probable states (persistent regimens) in the intertype game

Figure 2 depicts two important states of the memory of agents playing against opponents of different tag -intertype game- On the left simplex, everyone plays the strategy one-half, whereas on the right one every agent of one group (circles in the example) demands high against agents of the other group (squares), who consequently demand low.

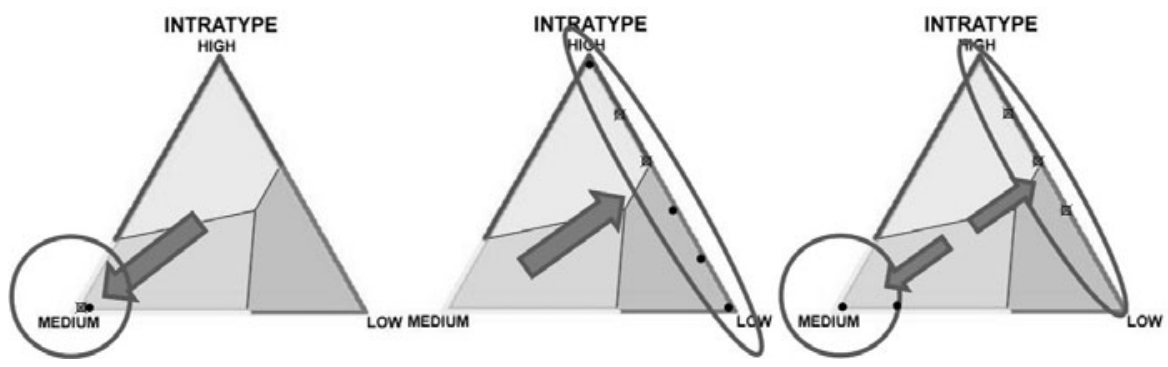

Fig. 3. Most probable states in the intratype game 
Figure 3 shows the three possible final states of the intratype interactions. In the first one (left), equity emerges within two groups (circles and squares). The second one (middle) represents a fractious state where both groups have members demanding simultaneously low and high. And finally, the third state (right) depicts a segregated situation in which equity emerges within one group (circles) whereas the other group (squares) plays inequality strategies.

\section{Other Persistent Regimes}

The results commented in the previous section confirm that the same six combinations of intertype and intratype persistent regimes pertaining to the global interaction game can also be obtained in the regular lattice game. Notwithstanding, when we run random intensive simulations in the spatial game we find that there is someone that needs a much higher time to converge to one of the mentioned regimens than the average time. This fact could suggest the appearance of some other basins of attraction beyond the mentioned combinations of states. This might imply that there are more situations where the transient dynamics of the system differs from the long run behavior of the system. A deeper insight into the tag spatial distribution puts forward two different types of situations (see Figure 4).

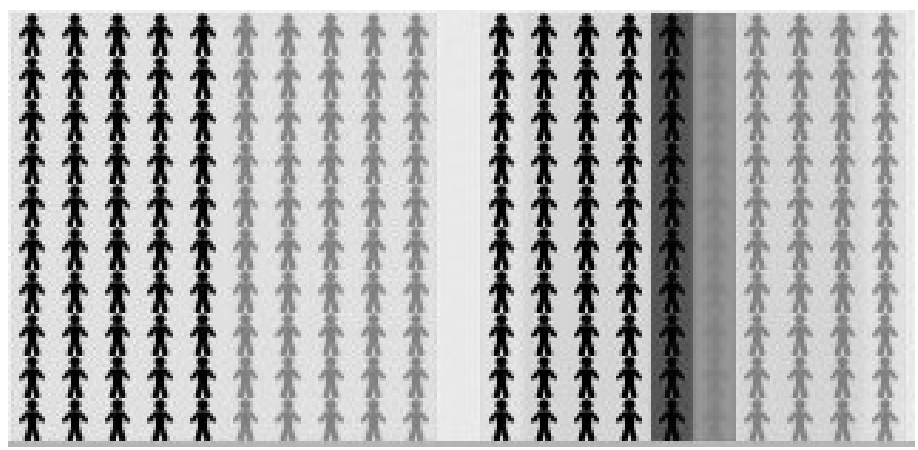

Fig. 4. The intertype plot (right) shows two different persistent regimens: one LH at the center and another MM at the right and left borders. Notice that the lattice is a torus.

A first case occurs when the particular distribution of tagged agents in the grid results in isolated areas of players with the same tag, without any contact between them. The behavior of disconnected frontiers of agents of the same type can produce different persistent regimens in each frontier. An extreme case of this effect is represented in Figure 4.

However, the other kind of situations is much more interesting. Now, the system state is characterized by non-disconnected clusters of agents -with the same tag- playing simultaneously different types of intertype coordination. The key difference with the previous case is the existence of an interconnection between the clusters.

The analyzed cases suggest that this last regime only appears when there is a strong community structure in the underlying network topology of the players, and that each 
regime corresponds to a different community. Our hypothesis is that the regimes obtained in the regular lattice version of the game are influenced by a mesoscale dimension between the individual and the complete population. In order to verify this intuition we have studied the community structure of an idealized case when this phenomenon frequently appears (see Figure 5).

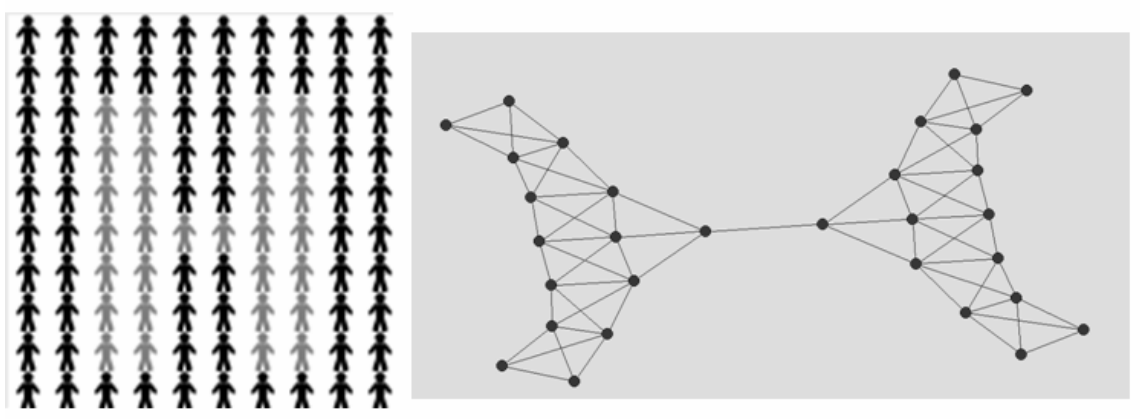

Fig. 5. We have analyzed the effects that appear in the stylized configuration showed on the left of the figure. On the right we represent the underlying interaction structure for the intratype game of grey-tagged players.

In order to find out the community structure of the network, we have used the Girvan-Newman algorithm [11] based on the iterative removal of the nodes with more betweenness. Our results depicted in Figure 6 show that, as predicted, the different regimes correspond to the partition of the network in communities detected by the algorithm.

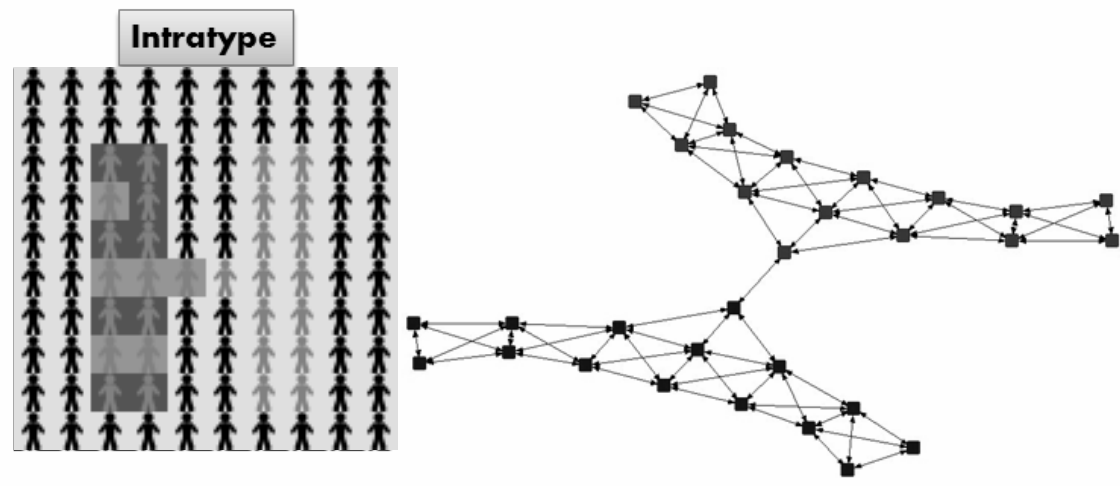

Fig. 6. On the left of the picture we can observe two different simultaneous persistent regimes in a connected population of agents with the same tag (h-shaped area). The different regimes correspond exactly to the different communities obtained in the underlying network of neighbors. 


\section{Conclusions}

In this work we have analyzed the Nash demand game played by a finite population of tagged agents situated in a regular lattice and playing as decision rule the best reply against the opponent's most frequent demand. Our results confirm that all the different regimes obtained in the global interaction model, including discriminatory norms, can emerge. Furthermore, computational simulations show that different persistent regimes can appear simultaneously within a group of agents with the same tag depending on the topology -especially the mesoscopic characteristics of the network-. Some results correspond to the trivial case, when there are disconnected clusters of players with the same tag, but also to other non-evident distributions of tagged agents in the grid. A preliminary study suggests that the community structure of the network of players with the same tag could be a strong predictor of these special regimes of the spatial version of the Nash demand game.

\section{Acknowledgments}

The authors have benefited from the financial support of the Spanish Ministry of Science and Technology (project TIN2008-06464-C03-02), of the Junta de Castilla y León (project BU034A08) and Caja Burgos (project 2009/00148/001 and project 2009/00199/001).

\section{References}

1. Axtell, R.L., Epstein, J.M., Young, H.P.: The Emergence of Classes in a Multi-Agent Bargaining Model. In: Durlauf, S.N., Young, H.P. (eds.) Social Dynamics. MIT Press, Washington (2004)

2. Galán, J.M., Izquierdo, L.R., Izquierdo, S.S., et al.: Errors and artefacts in agent-based modelling. Journal of Artificial Societies and Social Simulation 12, 1 (2009)

3. Izquierdo, L.R., Galán, J.M., Santos, J.I., del Olmo, R.: Modelado de sistemas complejos mediante simulación basada en agentes y mediante dinámica de sistemas. EMPIRIA Revista de Metodología de Ciencias Sociales 16, 85-112 (2008)

4. López-Paredes, A., Hernández, C.: Agent-based modelling in Natural Resource Management. Insisoc, Madrid (2008)

5. Walker, D.C., Hill, G., Smallwood, R.H., Southgate, J.: Agent-based computational modelling of wounded epithelial cell monolayers. IEEE Transactions on Nanobioscience 3, 153-163 (2004)

6. Araúzo-Araúzo, J.A., Galán-Ordax, J.M., Pajares-Gutiérrez, J., López-Paredes, A.: Gestión eficiente de carteras de proyectos. Propuesta de un sistema inteligente de soporte a la decisión para oficinas técnicas y empresas consultoras. DYNA Ingeniería e Industria 84, 761-772 (2009)

7. Coleman, J.S.: Foundations of social theory. Belknap Press of Harvard University Press, Cambridge (1994)

8. Galan, J.M., Izquierdo, L.R.: Appearances Can Be Deceiving: Lessons Learned ReImplementing Axelrod's 'Evolutionary Approach to Norms'. Journal of Artificial Societies and Social Simulation 8, 2 (2005) 
9. Poza, D., Villafáñez, F.A., Pajares, J.: Impact of Tag Recognition in Economic Decisions. In: Hernandez, C., Posada, M., López-Paredes, A. (eds.) Artificial Economics. The Generative Method in Economics. Springer, Heidelberg (2009)

10. Izquierdo, L.R., Izquierdo, S.S., Galán, J.M., Santos, J.I.: Techniques to understand computer simulations: Markov chain analysis. Journal of Artificial Societies and Social Simulation 12, 6 (2009)

11. Girvan, M., Newman, M.E.J.: Community structure in social and biological networks. Proceedings of the National Academy of Sciences of the United States of America 99, 7821-7826 (2002) 